\title{
MRI Predictors of Cognitive Training Outcomes
}

\author{
Ebru Baykara $^{1} \cdot$ Tanja Könen ${ }^{1} \cdot$ Kerstin Unger ${ }^{2} \cdot$ Julia Karbach $^{1}$ \\ Received: 17 December 2019 / Accepted: 28 August 2020 / Published online: 21 September 2020 \\ (C) The Author(s) 2020
}

\begin{abstract}
The prospect of improving or maintaining cognitive functioning has provoked a steadily increasing number of cognitive training interventions over the last years, especially for clinical and elderly populations. However, there are discrepancies between the findings of the studies. One of the reasons behind these heterogeneous findings is that there are vast inter-individual differences in how people benefit from the training and in the extent that training-related gains are transferred to other untrained tasks and domains. In this paper, we address the value of incorporating neural measures to cognitive training studies in order to fully understand the mechanisms leading to inter-individual differences in training gains and their generalizability to other tasks. Our perspective is that it is necessary to collect multimodal neural measures in the pre- and post-training phase, which can enable us to understand the factors contributing to successful training outcomes. More importantly, this understanding can enable us to predict who will benefit from different types of interventions, thereby allowing the development of individually tailored intervention programs.
\end{abstract}

Keywords Neuroimaging $\cdot$ Cognitive training $\cdot$ Cognitive plasticity $\cdot$ Neural plasticity $\cdot$ Individual differences

\section{Introduction}

Cognitive training has become increasingly popular (see Strobach and Karbach 2020, for a review) as the elderly population has rising life expectancy and therefore growing risk of cognitive and functional decline. Moreover, the cognitive demands for academic and occupational success are increasing with each generation. The main promise of cognitive training interventions is to induce lasting performance gains in cognitive domains that go beyond the practiced task and are relevant for daily functioning. Training-induced changes are thought to be triggered by a prolonged mismatch between situational demands and range of functions and performance an individual's cognitive system is able to support (Lövdén et al. 2010). This mismatch fosters adaptive structural brain changes (e.g., neurogenesis, synaptogenesis, long-term potentiation) that effectively increase the possible range of

Ebru Baykara

baykara@uni-landau.de

1 Department of Psychology, University of Koblenz-Landau, Landau/ Pfalz, Germany

2 Department of Psychology, Queens College, City University of New York, New York City, NY, USA cognitive performance to meet the altered environmental demands. Although the results of many training studies are promising, there is high variability across studies and individuals in such training-induced plastic changes (e.g., Katz et al. 2016, for a review), and even meta-analyses on the topic reveal conflicting conclusions (e.g., Kassai et al. 2019; MelbyLervåg and Hulme 2016 vs. Au et al. 2016; Karbach and Verhaeghen 2014; Nguyen et al. 2019). On the one hand, this reflects large differences between studies in terms of training type, training features, and target population (see Fig. 1, panel 3 ), but it also highlights large inter-individual differences in performance gains.

In recent years, these individual differences in traininginduced cognitive performance gains have attracted considerable scientific interest (e.g., Bürki et al. 2014; Karbach et al. 2017; Lövdén et al. 2012). According to the supply-demand mismatch model (Lövdén et al. 2010), the extent to which mismatch drives plastic changes depends on the current state of flexibility of the cognitive system. For instance, if environmental demands greatly exceed the existing functional capacity - as would be the case when asking a 4-year-old to maintain 7 digits in working memory-the impetus for change will be reduced. When the inter-individual variation is high, averaging across participants can be misleading (e.g., Moreau and Corballis 2018). It is now clear that the "one-fitsall" solutions are not working for cognitive training, and it is 


\begin{tabular}{|c|c|c|c|}
\hline Pretest & Intervention & Posttest & Follow-up \\
\hline $\begin{array}{l}\text { (1) Multimodal } \\
\text { Neuroimaging } \\
\text { Baseline individual differences in } \\
\text { structure and function of the } \\
\text { brain and their interactions }\end{array}$ & \multirow{3}{*}{$\begin{array}{l}\text { (3) Cognitive Training } \\
\text { Interventions may differ in } \\
\text { key features, such as: } \\
\text { - Training type (e.g., } \\
\text { process-based, strategy- } \\
\text { based, multi-domain) } \\
\text { - Target group } \\
\text { - Training features (e.g., } \\
\text { duration, intensity, } \\
\text { adaptivity, domain, } \\
\text { platform, setting) }\end{array}$} & $\begin{array}{l}\text { (4) Multimodal Neuroimaging } \\
\text { - Training-related structural and functional } \\
\text { changes } \\
\text { How do these changes interact? } \\
\text { Do these changes relate to cognitive changes? } \\
\text { Do these changes relate to baseline neural and } \\
\text { behavioral characteristics? }\end{array}$ & $\begin{array}{l}\text { (6) Multimodal Neuroimaging } \\
\text { - Longevity of structural and functional changes } \\
\text { che they related to the longevity of behavioral } \\
\text { ches } \\
\text { Are they related to to baseline neural and } \\
\text { behavioral characteristics? }\end{array}$ \\
\hline Prediction & & Immediate plasticity & Long-term plasticity \\
\hline $\begin{array}{l}\text { Baseline individual differences in } \\
\text { cognition, motivation, } \\
\text { personality etc. }\end{array}$ & & $\begin{array}{l}\text { (5) Behavioral Assessment } \\
\text { Training-related gains } \\
\text { Dransfer effects } \\
\text { Do these changes relate to neural changes? } \\
\text { behaves changes relat characteristics? baseline neural and }\end{array}$ & $\begin{array}{l}\text { (7) Behavioral Assessment } \\
\text { - Longevity of training and transfer gains } \\
\text { Are they related to the longevity of neural } \\
\text { changes? } \\
\text { - Are they related to to baseline neural and } \\
\text { behavioral characteristics? }\end{array}$ \\
\hline
\end{tabular}

Interaction of baseline characteristics, training-related change, and long-term outcomes

Fig. 1 Tentative model of a study design. We suggest including neuroimaging pre- and post-training as well as in the follow-up to be able to understand the mechanisms leading to cognitive training gain, generalization to unrelated tasks, and maintenance of these effects over longer periods of time

time to move towards individualized training programs (Colzato and Hommel 2016; Karbach and Unger 2014; Kliegel and Bürki 2012). In order to do that, we need to (1) determine which inter-individual differences lead to the variation in training-related outcomes and (2) understand the mechanisms leading to training gain and transfer.

In an attempt to identify individual characteristics that might influence the success of a training regimen, previous studies focused on age, sex, education, baseline cognitive performance, intelligence, personality, and motivation (e.g., Katz et al. 2016, for a review; see Fig. 1, panel 2). The results of these studies remain inconclusive. For instance, most studies agree that baseline cognitive performance is associated with training-related changes, but there is no agreement on the direction of this relation. Some reports found greater training-induced gains in individuals with higher baseline performance (Foster et al. 2017; Wiemers et al. 2019), whereas others concluded that individuals with low baseline performance benefit more because they have more room to improve (Jaeggi et al. 2011; Zinke et al. 2014). There is an increasing trend to combine basic demographic, psychometric, and behavioral measures with magnetic resonance imaging (MRI)based measures of brain morphological and functional characteristics to resolve these inconsistencies. The rationale is that brain markers are reliable indicators of the current functional organismic capacity, i.e., the possible range of cognitive performance. Neural predictors can be rather specific (e.g., hippocampal subfield volume) or more general (e.g., wholebrain functional connectivity patterns), depending on the complexity of the cognitive functions they are believed to support.
Moreover, direct assessment of training-induced change in brain structure and function has advanced the understanding of the mechanisms underlying cognitive performance increments. In this article, we give an overview of findings on brain structural and functional predictors of cognitive improvement as well as training-related brain changes. We discuss implications for future training research and address existing practical challenges.

\section{Brain Structural Factors Predicting Training-Induced Gain}

\section{Regional Volumes}

There are several studies showing that the volume of certain brain regions is associated with learning complex skills. The study of Erickson et al. (2010) with young adults revealed that the volume of striatum, but not hippocampus, predicted successful learning of a complex video game, which required coordination of various cognitive, motor, and perceptual systems. Similarly, another study (Basak et al. 2011) demonstrated in older adults that larger regional gray matter volumes (of the cerebellum, the dorsolateral prefrontal cortex, and the anterior cingulate cortex) predicted better learning rates in a realtime strategy video game. Using a standard dual-task paradigm, Verghese et al. (2016) found a correlation between the volume of the left dorsolateral prefrontal cortex (DLPFC) and multitasking training outcomes; however, the reported relation was negative, i.e. smaller volumes were associated with 
higher gains. Due to the diversity of methods used in these studies, a direct comparison is not possible and independent replications of the nature and direction of effects are needed.

According to the findings of Engvig et al. (2012b), pretraining hippocampal volumes were positively associated with memory improvements after a strategy-based cognitive training program in adults with memory complaints. Baseline hippocampal volumes were also found to predict improvements in mathematical skills after tutoring in primary-grade school children (Supekar et al. 2013). The researchers also investigated the effects of individual differences in behavioral measures on skill acquisition, and interestingly, they showed that neither pre-training IQ, working-memory performance, nor mathematical abilities predicted the performance gains. Findings of another study (Strangman et al. 2010) using memory strategy training and fully automated morphometric brain parcellation technique with traumatic brain injury patients revealed that the volumes of the hippocampus, the DLPFC, and the posterior parietal cortex were positively correlated with training-related memory improvement. However, another study (Peter et al. 2018) using memory strategy training found that the volume of entorhinal cortex rather than of the hippocampus was predictive of episodic memory improvement in patients with mild cognitive improvement. They also showed that baseline cognitive performance was not associated with memory improvement. Different segmentation and parcellation algorithms, different regions of interests, and different target populations may have contributed to these disparate findings.

Notably, not only regional volumes but also individual differences in cortical thickness can predict cognitive gains in older adults following a metamemory training program (Park et al. 2018). The authors investigated which brain characteristics can predict post-training cognitive improvement and showed the lower cortical thickness in parietal and occipital regions (cuneus, precuneus, posterior cingulate) was correlated with higher cognitive gains. Importantly, trainingrelated cognitive performance improvements in this study were measured using a battery of tests assessing verbal and non-verbal memory, language skills, short-term memory, and verbal fluency. Unfortunately, the use of a composite score did not allow conclusions as to whether the training improved all or only a subset of the above skills.

Taken together, the results demonstrate that brain morphology before cognitive training can predict how the trainees benefit from the training program (see Fig. 1, panel 1). Unfortunately, due to vast differences between the study protocols, analysis methods, and study populations, the studies do not tell a completely coherent story yet. Currently, most findings should be interpreted in the context of the given study and sample characteristics to avoid over-generalization. We can summarize that hippocampal volume has been associated with learning and memory improvement, and striatal volume was found to play a role when acquiring complex new skills such as the skills needed in video game playing (e.g., cognitive flexibility, integration and coordination of motor and perceptual information).

\section{Structural Connectivity}

Studies show that structural connectivity of the brain is related to cognitive function in healthy (Deary et al. 2006) and clinical populations (e.g., Baykara et al. 2016; Damoiseaux et al. 2009). Based on the following studies, it can be argued that the integrity of white matter tracts can be used to predict cognitive training outcomes.

Ray et al. (2017) investigated the relevance of microstructural integrity of several white matter regions for video game learning in younger and older adults. They found that white matter integrity in the fornix/stria terminalis was related to action game learning while white matter integrity in the cingulum/hippocampus was related to strategy game learning. Furthermore, they showed that although the participants' baseline cognitive abilities predicted general learning ability, they did not differentiate between different types of learning gains, whereas white matter integrity was sensitive to these differences. This demonstrates a differential predictive validity of brain measures vs. behavioral measures.

In their study, de Lange et al. (2016) demonstrated that in older participants as compared with younger participants, white matter structural integrity declined, especially in the anterior brain regions. More importantly, they showed that the baseline white matter integrity of the brain regions affected by aging, including the anterior corpus callosum, the left anterior thalamic radiation, and the right inferior fronto-occipital fasciculus, predicted the effects of memory training on memory performance in older adults.

In the above-mentioned multi-strategic metamemory training study, Park et al. (2018) investigated the structural connectivity in older adults and its association with cognitive improvements following training. They showed that white matter integrity in the right splenium of the corpus callosum predicted changes in a composite cognitive test score in the training group. Moreover, white matter anisotropy of right and left crura of fornix predicted memory enhancement specifically. Importantly, none of the baseline cognitive abilities predicted cognitive improvement, which shows the value of incorporating brain measures into cognitive training research. Similarly, Wolf et al. (2014) studied the association between structural integrity and transfer of training gains. They trained participants in logical reasoning and tested short-term and long-term transfer to fluid intelligence scores. The results of this study revealed that maintenance of transfer effects was associated with the integrity of the corpus callosum, and not with age or general intelligence. To be able to predict who will maintain training and transfer effects gives 
us the opportunity to properly select the target population and, for example, to adapt the intensity or duration of the training for those who have declined structural integrity.

The aforementioned studies show that structural integrity of the brain can provide valuable information on who can have a faster learning rate, who can benefit from different types of learning tasks, and who can show higher cognitive gains and transfer effects (see Fig. 1, panel 1). A higher integrity of white matter means more efficient neuronal conduction in the brain, which allows for effective information transfer (Kanai and Rees 2011). As several studies already showed, higher structural integrity also translates to better learning and better training outcomes.

\section{Brain Functional Factors Predicting Cognitive Gain}

Not only the structure but also the functional organization and connectivity can inform us about trainability potential. In this section, we review studies showing an association between baseline brain function and training-related gains.

\section{Resting-State Functional Connectivity}

MRI studies demonstrated a relation between the neural activation of brain networks and task performance, as well as learning performance (Baldassarre et al. 2012; VenturaCampos et al. 2013). Based on the assumption that learning performance is influenced by the connectivity patterns of intrinsic whole-brain networks, a study (Yamashita et al. 2015) investigated the relation between functional connections in the brain and subsequent learning. They showed that functional connections within the task-relevant networks predicted participants' learning ability of working memory tasks.

The following studies also focused on functional connectivity of whole-brain networks, since complex behavior is accepted to be a result of an interplay between different networks in the brain. Additionally, these studies used graph theory to quantify the brain's network organization and connectivity patterns. Graph theory is a mathematical model to quantify, illustrate, and analyze complex brain networks (structural and functional) and is widely used in neuroimaging research (for more information, see Bullmore and Sporns 2009; Sporns 2018). It has to be noted, however, that different types of connectivity analyses render differential results. While whole-brain connectivity is reasonably reliable, the reliability of individual resting-state connections is relatively low, though the application of multivariate prediction models can improve the reliability (cf. Sripada et al. 2020).

Several studies found that whole-brain modularity - a measure of balance between integration and segregation of brain networks - as calculated from resting-state functional MRI can predict gains related to cognitive interventions. The main finding is that higher brain network modularity is associated with better learning, higher training-related gains, and transfer (see Fig. 1, panel 1). These results have been established with patients with brain injury (Arnemann et al. 2015), older (Gallen et al. 2016), and younger adults (Baniqued et al. 2019), and following cognitive or physical training (Baniqued et al. 2018). The same principle is also valid in other types of learning such as motor learning: in their study, Mattar et al. (2018) found that a lower correlation between baseline motor and visual regions, in other words, more modular visual and motor networks, was predictive of faster learning.

The explanation of these findings is that modular networks at rest have overall high performance because they can adapt to the external demands faster (learn), and this reduces the brain's general wiring cost. It means modularity enables successful learning as it enables the brain to process information faster and adapt faster to changing environments (Ellefsen et al. 2015; Wig 2017; for a review of modularity as a marker of intervention-related plasticity, see Gallen and D'Esposito 2019).

\section{Task-Based fMRI}

Additionally, there are studies investigating task-based MRI to predict training gains. One example is a study with older adults (Heinzel et al. 2014) that examined the brain responses under different working memory (WM) loads (pre-training) and their correlation with cognitive gains resulting from WM training. Their conclusion was that older adults with activation patterns similar to young adults (lower activation at low WM load, and higher activation at high WM load) gained more from cognitive training. Furthermore, the pretraining activity of motor network has been also associated with training-related improvements in WM ability (Simmonite and Polk 2019). The authors discuss the relevance of motor network in terms of its relation to the ability of manipulating internal information.

In line with video game training studies (e.g., Erickson et al. 2010), Vo et al. (2011) showed that not only the volume but also the activity of striatum predicts learning success in a complex video game training. It is important to note, however, that not all studies found brain measures to be predictive of performance gains. In the abovementioned study, Heinzel et al. (2014) also investigated the predictive power of WMload-dependent functional connectivity and reported that there was no association between baseline connectivity and training outcome in the older sample. Considering the reliability issues related to task-based fMRI (Elliott et al. 2020; see "Practical challenges"), researchers should be cautious when interpreting their results (especially for prediction purposes) 
and not solely rely on task-based fMRI results for investigating individual differences.

The studies mentioned so far address the possibility of using neuroimaging methods to predict cognitive training gains (see Fig. 1, panel 1). The idea is that the baseline brain state of trainees (either the volume of relevant brain structures, the structural integrity of white matter or resting-state functional connectivity) has an influence on how they process information, i.e., how they learn and benefit from training (for an overview of the prediction studies please see Table 1).

\section{Training-Induced Neural Changes}

\section{Brain Structural Changes Following Cognitive Training}

There are different types of structural changes as reported in cognitive training studies: volume changes in cortical or subcortical structures, changes in cortical thickness, and in structural connectivity. Engvig et al. (2010) applied an intensive memory training program and found increases in cortical thickness in older adults. Furthermore, these cortical changes were correlated positively with the memory improvements observed after the training. The same group also revealed that the training had positive influence on white matter integrity in older adults (Engvig et al. 2012a). Lövdén et al. (2010) also reported increased white matter integrity in older adults after a multidomain (working memory, episodic memory, and perceptual speed) training program. Similarly, Takeuchi et al. (2010) investigated the effect of working memory training on structural connectivity and showed training-related positive changes in white matter integrity in regions that are involved in working memory processes. Additionally, this study also disclosed a positive correlation between changes in structural connectivity and the amount of working memory training, indicating a direct relation between the training and structural change. Furthermore, another study (Caeyenberghs et al. 2016) reported an increased structural (white matter) network connectivity, indicating greater global integration within a frontoparietal network following a working memory training. Accordingly, training-related improvements were associated with increased global efficiency, which provides an indication of how effectively information is integrated across the entire network.

On the other hand, despite these promising findings, some studies found no structural volume changes after an attention training (Mozolic et al. 2010) or multidomain training program (Adcock et al. 2020; Suo et al. 2016). Furthermore, some studies did not find changes in white matter integrity following a working memory (Fissler et al. 2017) or multidomain training program (Lampit et al. 2015). It is also important to keep in mind that not all studies directly investigated or reported the relation between the structural and cognitive changes. Some studies reported a positive association between neural and cognitive changes in gray matter (Engvig et al. 2010; Lampit et al. 2015) and in white matter (Engvig et al. 2012a; de Lange et al. 2017), whereas others did not (WM; Lövdén et al. 2010; Román et al. 2017). These inconsistent findings might be the results of the diversity of training programs and dosage, and further research is needed to clarify the factors leading to structural changes.

To conclude, a majority of the studies indicate that structural changes are relevant for measuring cognitive changes as a result of cognitive training (see Fig. 1, panel 4 and 5), and these brain changes could be used as biomarkers of neuroplasticity. Here, one cautionary note is needed. Single pre-post measures of brain volume can be associated with misleading results in cognitive training research. According to the expansion-renormalization model (Wenger et al. 2017), learning and skill acquisition can lead to a brain volume expansion in task-relevant areas due to an increase of, for example, neurons and synapses. However, this increase is usually followed by a selection process leading to a partial or even complete return to baseline volume (cf. Wenger et al. 2017). Thus, training-induced structural changes should be tracked across multiple time points during and after training and need to be complemented by functional assessments of cognitive performance to reveal a complete picture of their plasticity. As cognitive training involves skill acquisition (Gathercole et al. 2019), this model could apply to cognitive training-related structural plasticity. Empirical evidence for the model in this specific context is still needed, but it needs to be considered when interpreting changes in brain volume.

\section{Functional Brain Changes Following Cognitive Training}

In addition to the structural changes, several studies to date also revealed significant training-induced functional brain changes. Brehmer et al. (2011) investigated functional changes following an intense working memory training in older adults. Their results suggested an intervention-related increase in the neural efficiency in frontoparietal regions: participants receiving adaptive working memory training showed decreased brain activation while performing a difficult task as compared with the control group. Importantly, these changes were functionally relevant for the behavioral outcome, since the magnitude of the functional changes was correlated with the amount of cognitive improvement. In their study, Kirchhoff et al. (2012) trained older adults with semantic encoding strategies and showed that trained participants had increased encoding-related brain activity in regions associated with semantic memory (prefrontal and left temporal regions). Furthermore, training gains were correlated with the activity 
increases. Both of these studies revealed task-related activity changes in the brain following cognitive training, but the direction of the effects differed. Studies investigating trainingrelated changes in cortical activity report both increases (e.g., Kirchhoff et al. 2012; Nyberg et al. 2003; Olesen et al. 2004) and decreases (e.g., Brehmer et al. 2011; Miró-Padilla et al. 2019). On the other hand, other studies reveal a u-shaped pattern of changes following cognitive training in cortical (e.g., Hempel et al. 2004) and subcortical activation (e.g., Kühn et al. 2013). Accordingly, training can at first stimulate increases in activity, which are then followed by activity decreases suggesting more automatized processing or increased neural efficiency. These studies further highlight the importance of multiple measurements during and after cognitive training to yield a better understanding of the temporal dynamics of training-related neural changes.

In addition to task-based fMRI, some studies employed resting-state fMRI to reveal training-induced changes in functional connectivity. One study showed that working memory training with young adults resulted in increased functional connectivity between the key nodes of default mode network (DMN) (Takeuchi et al. 2013). A similar pattern of results was observed in older adults following a multidomain intervention (cognitive training and exercise): functional connectivity between parts of the DMN increased, and this increase was associated with improvements in cognitive performance ( $\mathrm{Li}$ et al. 2014). Chapman et al. (2015) demonstrated increases in functional connectivity within the DMN and central executive network (CEN) after a strategy-based training in older adults. Moreover, similar functional changes in brain networks were also reported in participants with mild cognitive impairment (MCI) and Alzheimer's disease (AD) (Barban et al. 2017).

Besides changes in functional connectivity within networks (e.g., DMN), there are also training-induced changes in connectivity patterns between networks. One example is the maintained segregation between DMN and CEN after a multidomain cognitive training in older adults, whereas the control group showed reduced segregation between these networks (Cao et al. 2016). Takeuchi et al. (2013) also showed decreases in the connectivity between a key DMN node and nodes of an external attention system following a working memory training in young adults. Taken together, there are conflicting results regarding the activity changes following cognitive training. One possibility is that these changes are dynamic and the studies failed to reveal the full temporal dynamics of those changes. Alternatively, the type of training is critical for the pattern of activity changes (Belleville et al. 2014) and the large methodological variability between studies results in conflicting conclusions. For the functional connectivity, the findings are more consistent: training results in increased connectivity within relevant networks, and decreased connectivity between networks, leading to increased segregation and increased modularity in the brain, which enables higher overall performance (Ellefsen et al. 2015).

There are a variety of structural and functional brain measures that can predict cognitive training-related gains, and cognitive training further induces structural and functional changes in the brain. In order to establish the clinical importance of these brain changes, studies should systematically investigate how baseline measures or post-training changes are linked to the observed behavioral changes.

Furthermore, studies also showed that structural, function$\mathrm{al}$, and behavioral changes occur on different time scales. Neural changes can occur in the absence of behavioral changes (Beauchamp et al. 2016), and functional changes can occur in the absence of structural changes (Lampit et al. 2015; Mozolic et al. 2010). Therefore, it is important to have multiple multimodal measurements to reveal and to understand the dynamic structural, functional, and behavioral changes and determine whether the training-related gains reflect plasticity, i.e., changes in functional capacity, rather than mere flexibility.

\section{Prediction of Transfer and Maintenance of Training Gains}

An important aim in cognitive training research is to predict generalization and long-term maintenance of training gains. Here, we suggest that investigating not only pre-training brain measures but also training-induced differences in brain structure, function, and connectivity can shed some light onto the underlying neural mechanisms of training-related changes, their transfer and maintenance (see Fig. 1, panels 4-7).

There are very few studies that investigated this particular relationship. In a study by Nikolaidis et al. (2014), participants played a video game that trained working memory, attention, and motor control skills. The findings revealed that functional activity changes in regions related to working memory (superior parietal lobe, precuneus and postcentral gyrus) predicted transfer to an untrained working memory task. Their results support the hypothesis that transfer is more likely to occur if training and transfer tasks share the same underlying neural mechanisms (Jonides 2004). This notion is also supported by the study of Dahlin et al. (2008). In their study, they showed that working memory training gains transferred only to an untrained task that activated the same brain region (striatum) as the training task.

The research into maintenance effects is even scarcer, since many studies do not include a follow-up measurement. One study (Parisi et al. 2014) investigated the effects of a cognitive training program (including attention, information processing, and executive function training modules) in multiple sclerosis patients. The results revealed that changes in the resting-state functional connectivity (from pre- to post-training) in the 
default mode network and of the anterior cingulum were associated with cognitive performance (attention and executive functions) measured 6 months after the training.

These findings support the idea that cognitive trainingrelated neural changes can indeed predict transfer to untrained tasks and even maintenance of training gains. Thus, cognitive neuroscience approach can offer an understanding of patterns of brain activity, brain networks, and anatomical structures that modulate transfer and maintenance. It is particularly desirable to have a marker that can tell before the training who is going to show sustained benefits.

\section{Practical Challenges}

Systematically incorporating neuroimaging into cognitive training research makes sense on a theoretical level; however, there are practical challenges. One of the biggest challenges is the time and financial cost of using neuroimaging. However, progress in understanding cognitive training-related gains and what makes a successful intervention has been relatively slow. This mainly relates to the lack of understanding of neural changes underlying cognitive plasticity. Therefore, many research teams working on cognitive interventions are already emphasizing the importance of neuroimaging in the cognitive training field (e.g., Belleville and Bherer 2012; Brehmer et al. 2014; Taya et al. 2015).

Additionally, there are technical challenges to overcome: using different neuroimaging protocols and modalities might make it difficult to compare findings from different studies with small sample sizes (and practically, due to the cost of neuroimaging, studies are very often severely underpowered even though large samples would be necessary particularly to analyze individual differences in performance gains and brain changes). As mentioned before, an important issue in training research is the retest reliability of MRI-based measures. While it is beyond the scope of this review to discuss the details of the reliability problem, there are methodological papers addressing this issue for different modalities and data analysis methods (e.g., brain volumes, Schnack et al. 2004; fMRI and functional connectivity, Dubois and Adolphs 2016; Noble et al. 2019; Shah et al. 2016; network neuroscience, Bassett and Sporns 2017; resting-state fMRI, Zuo and Xing 2014; structural connectivity; Buchanan et al. 2014; Roine et al. 2019). Although most of the methods used in the studies mentioned here have sufficient reliability, a recent meta-analysis reported that task-fMRI measures have poor reliability and therefore are unsuitable for investigating individual differences (Elliott et al. 2020). The authors recommend using multivariate methods, validating results in independent samples, and combining task-based data with resting-state fMRI to increase the value and reliability of existing datasets.

\section{Concluding Remarks and Future Directions}

This review presents preliminary but promising evidence suggesting that baseline brain characteristics can be employed to predict cognitive training gains. Furthermore, inter-individual neural differences at baseline and change induced by the training could be associated with transfer and maintenance of these gains. Most of the studies incorporating neuroimaging methods into cognitive training research investigated training-induced changes and not the predictive value for training gains, transfer, and maintenance. With this review, we want to encourage researchers to look at the brain from this perspective - as is already done in clinical research-and approach their data with new questions.

Tools from graph theory have been widely used to analyze the brain as a complex network and to explore and quantify brain-behavior relations (Sporns 2014). It is now possible to quantitatively synthesize data from different studies (e.g., Duda and Sweet 2019) and to build better neuromarkers by using multivariate, predictive models and by integrating multimodal data (Scheinost et al. 2019; Woo et al. 2017). These methods are already applied in various studies to predict behavior (e.g., disease state, cognitive status, response to treatment) with promising results (see Sui et al. 2020). It is time that the cognitive training field takes advantage of the new era, too. Ideally, we would invest in establishing more multi-centered collaborations and in designing projects with standardized multimodal imaging (structural and functional MRI) and standardized training protocols. It makes sense to have multiple measurement points (pre, during, post training and several follow-ups), and it is particularly important to assess the relation between brain alterations and behavior changes to emphasize the meaningfulness and relevance of neural changes. This can be the next step to increase our understanding of how the brain responds to training, how different measures (e.g., structure and function) interact with each other influencing training outcomes, and it may provide the basis for designing informed interventions.

Incorporating neural measures systematically into cognitive training research can help us to understand the brain mechanisms facilitating learning and to determine brain characteristics that enable transfer and long-term maintenance of changes induced by cognitive training (see Fig. 1). Identifying the brain characteristics predicting how individuals benefit from different types of cognitive training would allow selecting the right training program, tailoring training 


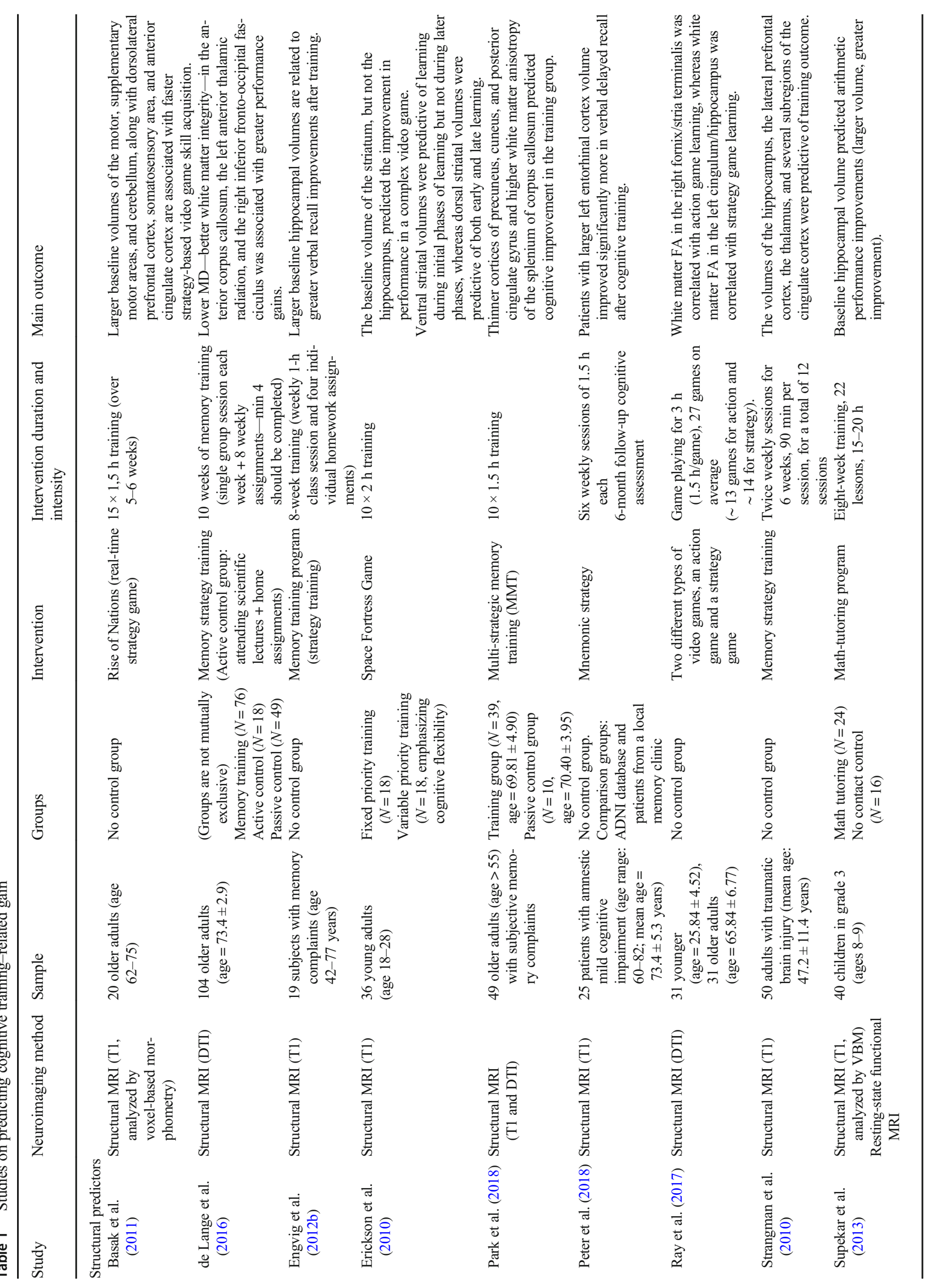




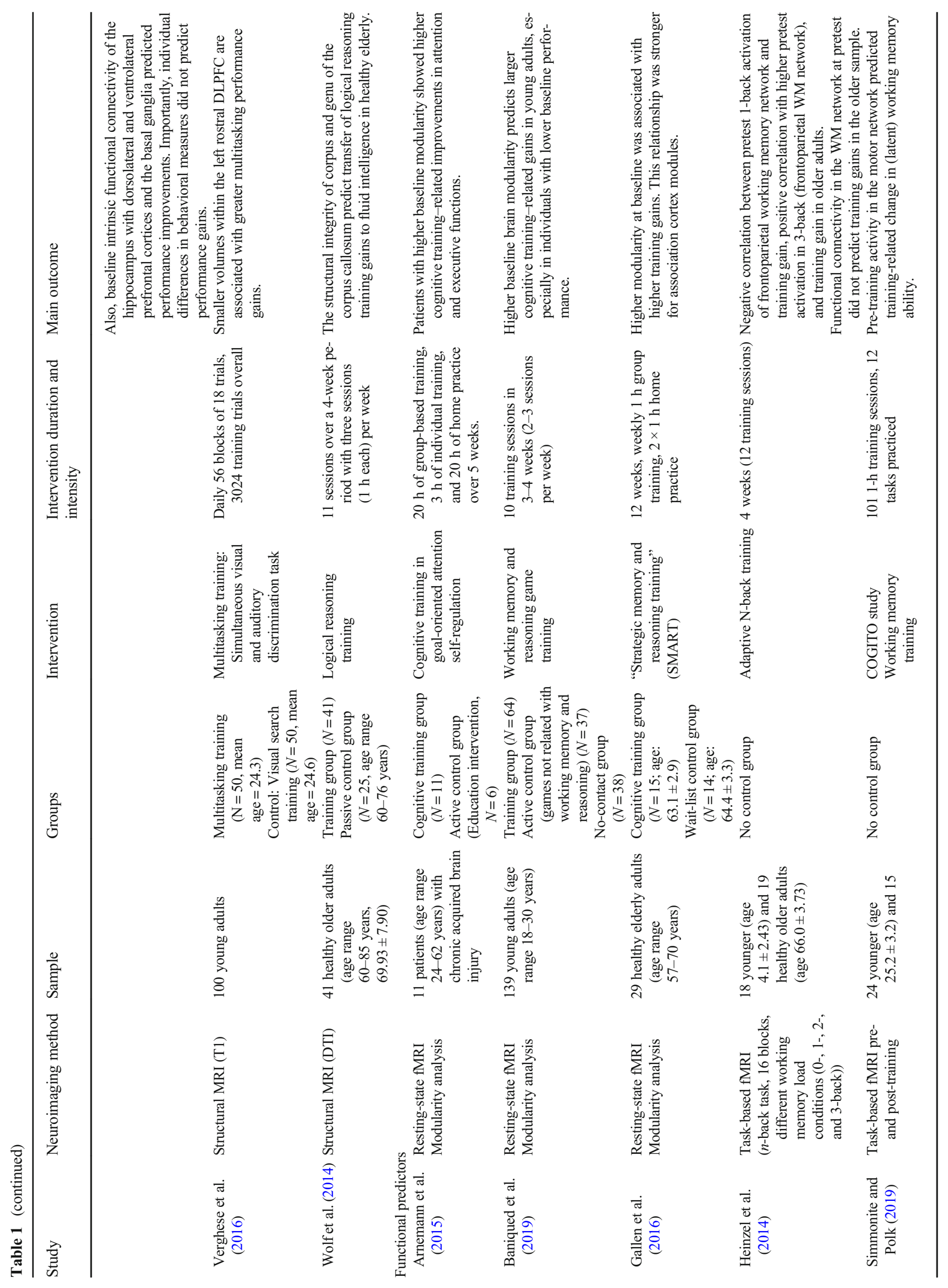




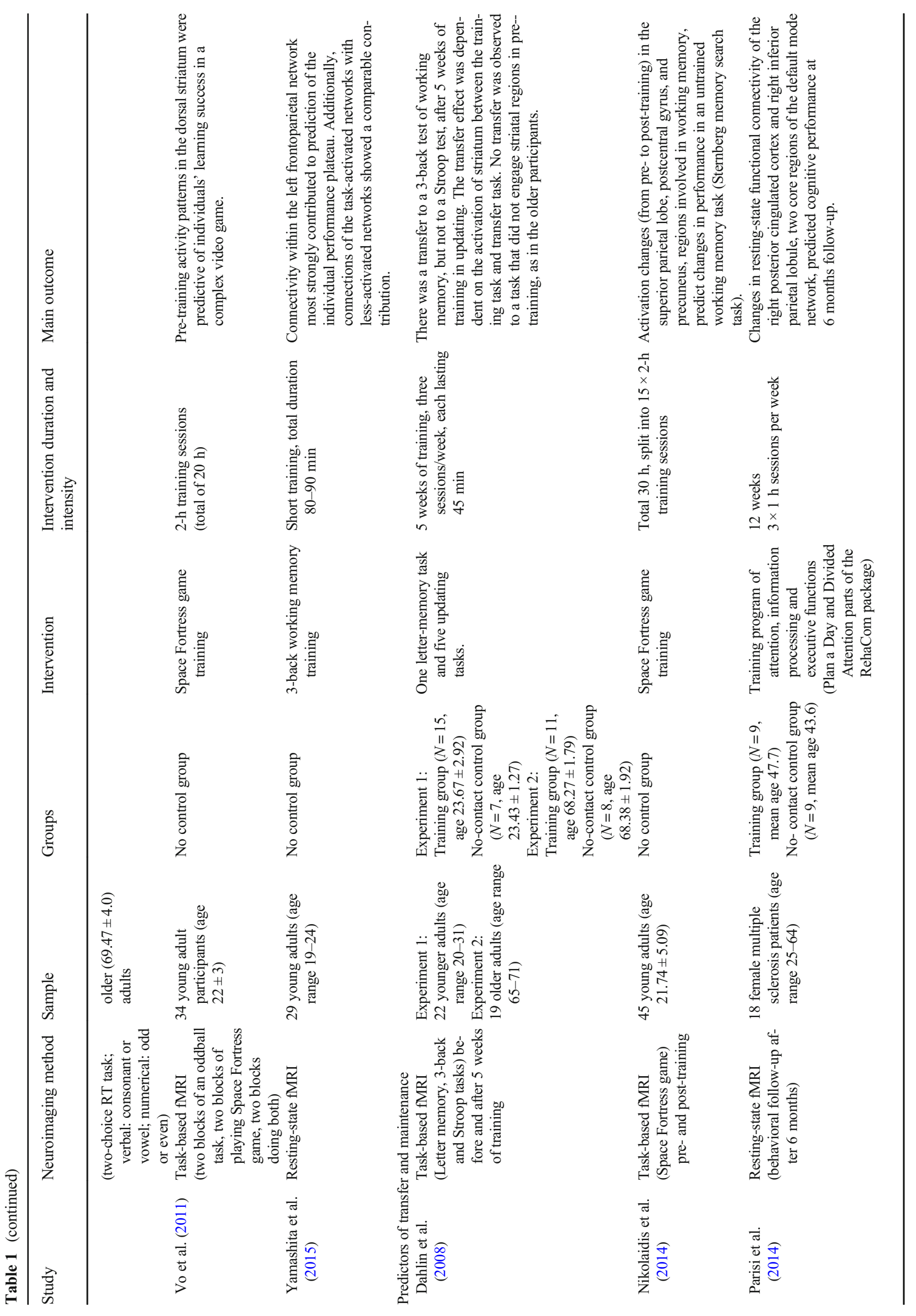


parameters (e.g., the type, duration, and intensity) to individual needs and ultimately to enhance the benefits of cognitive interventions.

Funding Open Access funding provided by Projekt DEAL.

\section{Compliance with Ethical Standards}

Conflict of Interest The authors declare that there is no conflict of interest.

Open Access This article is licensed under a Creative Commons Attribution 4.0 International License, which permits use, sharing, adaptation, distribution and reproduction in any medium or format, as long as you give appropriate credit to the original author(s) and the source, provide a link to the Creative Commons licence, and indicate if changes were made. The images or other third party material in this article are included in the article's Creative Commons licence, unless indicated otherwise in a credit line to the material. If material is not included in the article's Creative Commons licence and your intended use is not permitted by statutory regulation or exceeds the permitted use, you will need to obtain permission directly from the copyright holder. To view a copy of this licence, visit http://creativecommons.org/licenses/by/4.0/.

\section{References}

Adcock, M., Fankhauser, M., Post, J., Lutz, K., Zizlsperger, L., Luft, A. R., et al. (2020). Effects of an in-home multicomponent Exergame training on physical functions, cognition, and brain volume of older adults: a randomized controlled trial. Frontiers in Medicine, 6, 321.

Arnemann, K. L., Chen, A. J.-W., Novakovic-Agopian, T., Gratton, C., Nomura, E. M., \& D'Esposito, M. (2015). Functional brain network modularity predicts response to cognitive training after brain injury. Neurology, 84(15), 1568-1574. https://doi.org/10.1212/WNL. 0000000000001476.

Au, J., Buschkuehl, M., Duncan, G. J., \& Jaeggi, S. M. (2016). There is no convincing evidence that working memory training is NOT effective: a reply to Melby-Lervåg and Hulme (2015). Psychonomic Bulletin and Review, 23(1), 331-337. https://doi.org/10.3758/ s13423-015-0967-4.

Baldassarre, A., Lewis, C. M., Committeri, G., Snyder, A. Z., Romani, G. L., \& Corbetta, M. (2012). Individual variability in functional connectivity predicts performance of a perceptual task. Proceedings of the National Academy of Sciences, 109(9), 3516-3352. https://doi. org/10.1073/pnas.1113148109.

Baniqued, P. L., Gallen, C. L., Kranz, M. B., Kramer, A. F., \& D'Esposito, M. (2019). Brain network modularity predicts cognitive training-related gains in young adults. Neuropsychologia, 131, 205215. https://doi.org/10.1016/j.neuropsychologia.2019.05.021.

Baniqued, P. L., Gallen, C. L., Voss, M. W., Burzynska, A. Z., Wong, C. N., Cooke, G. E., et al. (2018). Brain network modularity predicts exercise-related executive function gains in older adults. Frontiers in Aging Neuroscience, 9, 426. https://doi.org/10.3389/fnagi.2017. 00426.

Barban, F., Mancini, M., Cercignani, M., Adriano, F., Perri, R., Annicchiarico, R., et al. (2017). A pilot study on brain plasticity of functional connectivity modulated by cognitive training in mild Alzheimer's disease and mild cognitive impairment. Brain Sciences, 7, 50. https://doi.org/10.3390/brainsci7050050.

Basak, C., Voss, M. W., Erickson, K. I., Boot, W. R., \& Kramer, A. F. (2011). Regional differences in brain volume predict the acquisition of skill in a complex real-time strategy videogame. Brain and Cognition, 76(3), 407-414. https://doi.org/10.1016/j.bandc.2011. 03.017.

Bassett, D. S., \& Sporns, O. (2017). Network neuroscience. Nature Neuroscience, 20(3), 353-364.

Baykara, E., Gesierich, B., Adam, R., Tuladhar, A. M., Biesbroek, J. M., Koek, H. L., ... Duering, M. (2016). A novel imaging marker for small vessel disease based on skeletonization of white matter tracts and diffusion histograms. Annals of Neurology, 80(4), 581-592. https://doi.org/10.1002/ana.24758.

Beauchamp, K. G., Kahn, L. E., \& Berkman, E. T. (2016). Does inhibitory control training transfer?: behavioral and neural effects on an untrained emotion regulation task. Social Cognitive and Affective Neuroscience, 11(9), 1374-1382.

Belleville, S., \& Bherer, L. (2012). Biomarkers of cognitive training effects in aging. Current Translational Geriatrics and Experimental Gerontology Reports, 1(2), 104-110. https://doi.org/ 10.1007/s13670-012-0014-5.

Belleville, S., Mellah, S. de Boysson, C., Demonet, J-F., Bier, B., Chao, L (2014) The Pattern and Loci of Training-Induced Brain Changes in Healthy Older Adults Are Predicted by the Nature of the Intervention. PLoS ONE 9 (8): 102710

Brehmer, Y., Kalpouzos, G., Wenger, E., \& Lövdén, M. (2014). Plasticity of brain and cognition in older adults. Psychological Research, 78(6), 790-802. https://doi.org/10.1007/s00426-014-0587-z.

Brehmer, Y., Rieckmann, A., Bellander, M., Westerberg, H., Fischer, H., \& Bäckman, L. (2011). Neural correlates of training-related working-memory gains in old age. NeuroImage, 58(4), 1110-1120. https://doi.org/10.1016/j.neuroimage.2011.06.079.

Buchanan, C. R., Pernet, C. R., Gorgolewski, K. J., Storkey, A. J., \& Bastin, M. E. (2014). Test-retest reliability of structural brain networks from diffusion MRI. Neuroimage, 86, 231-243.

Bullmore, E., \& Sporns, O. (2009). Complex brain networks: graph theoretical analysis of structural and functional systems. Nature Reviews Neuroscience, 10(3), 186-198. https://doi.org/10.1038/ nrn2575.

Bürki, C. N., Ludwig, C., Chicherio, C., \& de Ribaupierre, A. (2014). Individual differences in cognitive plasticity: an investigation of training curves in younger and older adults. Psychological Research, 78(6), 821-835. https://doi.org/10.1007/s00426-0140559-3.

Caeyenberghs, K., Metzler-Baddeley, C., Foley, S., \& Jones, D. K. (2016). Dynamics of the human structural connectome underlying working memory training. Journal of Neuroscience, 36(14), 40564066.

Cao, W., Cao, X., Hou, C., Li, T., Cheng, Y., Jiang, L., ... Yao, D. (2016). Effects of cognitive training on resting-state functional connectivity of default mode, salience, and central executive networks. Frontiers in Aging Neuroscience, 8, 70. https://doi.org/10.3389/ fnagi.2016.00070.

Chapman, S. B., Aslan, S., Spence, J. S., Hart, J. J., Bartz, E. K., Didehbani, N., ... Lu, H. (2015). Neural mechanisms of brain plasticity with complex cognitive training in healthy seniors. Cerebral Cortex, 25(2), 396-405. https://doi.org/10.1093/cercor/bht234.

Colzato, L. S., \& Hommel, B. (2016). The future of cognitive training. In Cognitive training (pp. 201-211). Springer, Cham.

Dahlin, E., Neely, A. S., Larsson, A., Bäckman, L., \& Nyberg, L. (2008). Transfer of learning after updating training mediated by the striatum. Science, 320(5882), 1510-1512. https://doi.org/10.1126/science. 1155466.

Damoiseaux, J. S., Smith, S. M., Witter, M. P., Sanz-Arigita, E. J., Barkhof, F., Scheltens, P., ... Rombouts, S. A. R. B. (2009). White matter tract integrity in aging and Alzheimer's disease. Human Brain Mapping, 30(4), 1051-1059. https://doi.org/10. 1002/hbm.20563. 
de Lange, A. M. G., Bråthen, A. C. S., Grydeland, H., Sexton, C., Johansen-Berg, H., Andersson, J. L. R., ... Walhovd, K. B. (2016). White-matter integrity as a marker for cognitive plasticity in aging. Neurobiology of Aging, 47, 74-82. https://doi.org/10.1016/ j.neurobiolaging.2016.07.007.

de Lange, A. M. G., Bråthen, A. C. S., Rohani, D. A., Grydeland, H., Fjell, A. M., \& Walhovd, K. B. (2017). The effects of memory training on behavioral and microstructural plasticity in young and older adults. Human Brain Mapping, 38(11), 5666-5680.

Deary, I. J., Bastin, M. E., Pattie, A., Clayden, J. D., Whalley, L. J., Starr, J. M., \& Wardlaw, J. M. (2006). White matter integrity and cognition in childhood and old age. Neurology, 66(4), 505-512. https:// doi.org/10.1212/01.wnl.0000199954.81900.e2.

Dubois, J., \& Adolphs, R. (2016). Building a science of individual differences from fMRI. Trends in Cognitive Sciences, 20(6), 425-443.

Duda, B. M., \& Sweet, L. H. (2019). Functional brain changes associated with cognitive training in healthy older adults: a preliminary ALE meta-analysis. Brain Imaging and Behavior, 1-16.

Ellefsen, K. O., Mouret, J.-B., \& Clune, J. (2015). Neural modularity helps organisms evolve to learn new skills without forgetting old skills. PLoS Computational Biology, 11(4), e1004128. https://doi. org/10.1371/journal.pcbi.1004128.

Elliott, M. L., Knodt, A. R., Ireland, D., Morris, M. L., Poulton, R., Ramrakha, S., ... \& Hariri, A. R. (2020). What is the test-retest reliability of common task-functional MRI measures? New Empirical Evidence and a Meta-Analysis. Psychological Science, 0956797620916786.

Engvig, A., Fjell, A. M., Westlye, L. T., Moberget, T., Sundseth, O., Larsen, V. A., \& Walhovd, K. B. (2010). Effects of memory training on cortical thickness in the elderly. NeuroImage, 52(4), 1667-1676. https://doi.org/10.1016/j.neuroimage.2010.05.041.

Engvig, A., Fjell, A. M., Westlye, L. T., Moberget, T., Sundseth, Ø., Larsen, V. A., \& Walhovd, K. B. (2012a). Memory training impacts short-term changes in aging white matter: a longitudinal diffusion tensor imaging study. Human Brain Mapping, 33(10), 2390-2406. https://doi.org/10.1002/hbm.21370.

Engvig, A., Fjell, A. M., Westlye, L. T., Skaane, N. v., Sundseth, Ø., \& Walhovd, K. B. (2012b). Hippocampal subfield volumes correlate with memory training benefit in subjective memory impairment. NeuroImage, 61(1). https://doi.org/10.1016/j.neuroimage.2012.02. 072.

Erickson, K. I., Boot, W. R., Basak, C., Neider, M. B., Prakash, R. S., Voss, M. W., ... Kramer, A. F. (2010). Striatal volume predicts level of video game skill acquisition. Cerebral Cortex, 20(11), 25222530. https://doi.org/10.1093/cercor/bhp293.

Fissler, P., Müller, H. P., Küster, O. C., Laptinskaya, D., Thurm, F., Woll, A., et al. (2017). No evidence that short-term cognitive or physical training programs or lifestyles are related to changes in white matter integrity in older adults at risk of dementia. Frontiers in Human Neuroscience, 11, 110.

Foster, J. L., Harrison, T. L., Hicks, K. L., Draheim, C., Redick, T. S., \& Engle, R. W. (2017). Do the effects of working memory training depend on baseline ability level? Journal of Experimental Psychology: Learning, Memory, and Cognition, 43(11), 16771689. https://doi.org/10.1037/xlm0000426.

Gallen, C. L., Baniqued, P. L., Chapman, S. B., Aslan, S., Keebler, M., Didehbani, N., \& D'Esposito, M. (2016). Modular brain network organization predicts response to cognitive training in older adults. PLoS One, 11(12), e0169015. https://doi.org/10.1371/journal.pone. 0169015 .

Gallen, C. L., \& D'Esposito, M. (2019). Brain modularity: a biomarker of intervention-related plasticity. Trends in Cognitive Sciences, 23(4), 293-304. https://doi.org/10.1016/j.tics.2019.01.014.

Gathercole, S. E., Dunning, D. L., Holmes, J., \& Norris, D. (2019). Working memory training involves learning new skills. Journal of Memory and Language, 105, 19-42.
Heinzel, S., Lorenz, R. C., Brockhaus, W.-R., Wustenberg, T., Kathmann, N., Heinz, A., \& Rapp, M. A. (2014). Working memory load-dependent brain response predicts behavioral training gains in older adults. The Journal of Neuroscience, 34(4), 1224-1233. https://doi.org/10.1523/jneurosci.2463-13.2014.

Hempel, A., Giesel, F. L., Garcia Caraballo, N. M., Amann, M., Meyer, H., Wüstenberg, T., et al. (2004). Plasticity of cortical activation related to working memory during training. American Journal of Psychiatry, 161(4), 745-747.

Jaeggi, S. M., Buschkuehl, M., Jonides, J., \& Shah, P. (2011). Short- and long-term benefits of cognitive training. Proceedings of the National Academy of Sciences, 108(25), 10081-10086. https://doi.org/10. 1073/pnas.1103228108.

Jonides, J. (2004). How does practice makes perfect? Nature Neuroscience, 7(1), 10-11.

Kanai, R., \& Rees, G. (2011). The structural basis of inter-individual differences in human behaviour and cognition. Nature Reviews Neuroscience, 12, 231-242. https://doi.org/10.1038/nrn3000.

Karbach, J., Könen, T., \& Spengler, M. (2017). Who benefits the most? Individual differences in the transfer of executive control training across the lifespan. Journal of Cognitive Enhancement, 1(4), 394405. https://doi.org/10.1007/s41465-017-0054-z.

Karbach, J., \& Unger, K. (2014). Executive control training from middle childhood to adolescence. Frontiers in Psychology, 5, 1-14. https:// doi.org/10.3389/fpsyg.2014.00390.

Karbach, J., \& Verhaeghen, P. (2014). Making working memory work: a meta-analysis of executive-control and working memory training in older adults. Psychological Science, 25, 2027-2037. https://doi.org/ 10.1177/0956797614548725.

Kassai, R., Futo, J., Demetrovics, Z., \& Takacs, Z. K. (2019). A metaanalysis of the experimental evidence on the near- and far-transfer effects among children's executive function skills. Psychological Bulletin, 145(2). https://doi.org/10.1037/bul0000180.

Katz, B., Jones, M. R., Shah, P., Buschkuehl, M., \& Jaeggi, S. M. (2016). Individual differences and motivational effects. In T. Strobach \& J. Karbach (Eds.), Cognitive training: an overview of features and applications (pp. 157-166). Springer International Publishing. https://doi.org/10.1007/978-3-319-42662-4_15.

Kirchhoff, B. A., Anderson, B. A., Barch, D. M., \& Jacoby, L. L. (2012). Cognitive and neural effects of semantic encoding strategy training in older adults. Cerebral Cortex, 22(4), 788-799. https://doi.org/10. 1093/cercor/bhr129.

Kliegel, M., \& Bürki, C. (2012). Memory training interventions require a tailor-made approach: commentary on McDaniel and Bugg. Journal of Applied Research in Memory and Cognition, 1, 58-60. https:// doi.org/10.1016/j.jarmac.2012.01.002.

Kühn, S., Schmiedek, F., Noack, H., Wenger, E., Bodammer, N. C., Lindenberger, U., \& Lövden, M. (2013). The dynamics of change in striatal activity following updating training. Human Brain Mapping, 34(7), 1530-1541.

Lampit, A., Hallock, H., Suo, C., Naismith, SL, Valenzuela, Michael. (2015) Cognitive training-induced short-term functional and long-term structural plastic change is related to gains in global cognition in healthy older adults: a pilot study. Frontiers in Aging Neuroscience 7

Li, R., Zhu, X., Yin, S., Niu, Y., Zheng, Z., Huang, X., ... Li, J. (2014). Multimodal intervention in older adults improves resting-state functional connectivity between the medial prefrontal cortex and medial temporal lobe. Frontiers in Aging Neuroscience, 6:39. https://doi. org/10.3389/fnagi.2014.00039.

Lövdén, M., Bäckman, L., Lindenberger, U., Schaefer, S., \& Schmiedek, F. (2010). A theoretical framework for the study of adult cognitive plasticity. Psychological Bulletin, 136, 659-676. https://doi.org/10. 1037/a0020080.

Lövdén, M., Bodammer, N. C., Kühn, S., Kaufmann, J., Schütze, H., Tempelmann, C., ... Lindenberger, U. (2010). Experience- 
dependent plasticity of white-matter microstructure extends into old age. Neuropsychologia, 48(13), 3878-3883. https://doi.org/10. 1016/j.neuropsychologia.2010.08.026.

Lövdén, M., Brehmer, Y., Li, S.-C., \& Lindenberger, U. (2012). Training-induced compensation versus magnification of individual differences in memory performance. Frontiers in Human Neuroscience, 6, 1-14. https://doi.org/10.3389/fnhum.2012.00141.

Mattar, M. G., Wymbs, N. F., Bock, A. S., Aguirre, G. K., Grafton, S. T., \& Bassett, D. S. (2018). Predicting future learning from baseline network architecture. NeuroImage, 172, 107-117. https://doi.org/ 10.1016/j.neuroimage.2018.01.037.

Melby-Lervåg, M., \& Hulme, C. (2016). There is no convincing evidence that working memory training is effective: a reply to Au et al. (2014) and Karbach and Verhaeghen (2014). Psychonomic Bulletin \& Review, 23(1). https://doi.org/10.3758/s13423-015-0862-z.

Miró-Padilla, A., Bueichekú, E., Ventura-Campos, N., Flores-Compañ, M. J., Parcet, M. A., \& Ávila, C. (2019). Long-term brain effects of N-back training: an fMRI study. Brain Imaging and Behavior, 13(4), 1115-1127.

Moreau, D., \& Corballis, M. C. (2018). When averaging goes wrong: the case for mixture model estimation in psychological science. Journal of Experimental Psychology: General. https://doi.org/10.1037/ xge 0000504

Mozolic, J. L., Hayaska, S., \& Laurienti, P. J. (2010). A cognitive training intervention increases resting cerebral blood flow in healthy older adults. Frontiers in Human Neuroscience, 4, 16.

Nguyen, L., Murphy, K., \& Andrews, G. (2019). Immediate and longterm efficacy of executive functions cognitive training in older adults: a systematic review and meta-analysis. Psychological Bulletin, 145, 698-733. https://doi.org/10.1037/bul0000196.

Nikolaidis, A., Voss, M. W., Lee, H., Vo, L. K., \& Kramer, A. F. (2014). Parietal plasticity after training with a complex video game is associated with individual differences in improvements in an untrained working memory task. Frontiers in Human Neuroscience, 8, 169.

Noble, S., Scheinost, D., Constable, R.T. (2019) A decade of test-retest reliability of functional connectivity: A systematic review and metaanalysis. NeuroImage 203:116157

Nyberg, L., Sandblom, J., Jones, S., Neely, A. S., Petersson, K. M., Ingvar, M., \& Bäckman, L. (2003). Neural correlates of trainingrelated memory improvement in adulthood and aging. Proceedings of the National Academy of Sciences, 100(23), 13728-13733.

Olesen, P. J., Westerberg, H., \& Klingberg, T. (2004). Increased prefrontal and parietal activity after training of working memory. Nature Neuroscience, 7(1), 75-79.

Parisi, L., Rocca, M. A., Mattioli, F., Copetti, M., Capra, R., Valsasina, P., ... \& Filippi, M. (2014). Changes of brain resting state functional connectivity predict the persistence of cognitive rehabilitation effects in patients with multiple sclerosis. Multiple Sclerosis Journal, 20(6), 686-694.

Park, S., Ryu, S.-H., Yoo, Y., Yang, J.-J., Kwon, H., Youn, J.-H., ... Lee, J.-Y. (2018). Neural predictors of cognitive improvement by multistrategic memory training based on metamemory in older adults with subjective memory complaints. Scientific Reports, 8:1095. https://doi.org/10.1038/s41598-018-19390-2.

Peter, J., Schumacher, L. V., Landerer, V., Abdulkadir, A., Kaller, C. P., Lahr, J., \& Klöppel, S. (2018). Biological factors contributing to the response to cognitive training in mild cognitive impairment. Journal of Alzheimer's Disease, 61(1), 333-345.

Ray, N. R., O'Connell, M. A., Nashiro, K., Smith, E. T., Qin, S., \& Basak, C. (2017). Evaluating the relationship between white matter integrity, cognition, and varieties of video game learning. Restorative Neurology and Neuroscience, 35(5), 437-456. https:// doi.org/10.3233/RNN-160716

Roine, T., Jeurissen, B., Perrone, D., Aelterman, J., Philips, W., Sijbers, J., \& Leemans, A. (2019). Reproducibility and intercorrelation of graph theoretical measures in structural brain connectivity networks. Medical Image Analysis, 52, 56-67.

Román, F. J., Iturria-Medina, Y., Martínez, K., Karama, S., Burgaleta, M., Evans, A. C., et al. (2017). Enhanced structural connectivity within a brain sub-network supporting working memory and engagement processes after cognitive training. Neurobiology of Learning and Memory, 141, 33-43.

Scheinost, D., Noble, S., Horien, C., Greene, A. S., Lake, E. M., Salehi, M., et al. (2019). Ten simple rules for predictive modeling of individual differences in neuroimaging. NeuroImage, 193, 35-45.

Schnack, H. G., van Haren, N. E., Hulshoff Pol, H. E., Picchioni, M., Weisbrod, M., Sauer, H., ... \& Kahn, R. S. (2004). Reliability of brain volumes from multicenter MRI acquisition: a calibration study. Human Brain Mapping, 22(4), 312-320.

Shah, L. M., Cramer, J. A., Ferguson, M. A., Birn, R. M., \& Anderson, J. S. (2016). Reliability and reproducibility of individual differences in functional connectivity acquired during task and resting state. Brain and behavior, 6(5), e00456.

Simmonite, M., \& Polk, T. A. (2019). Independent components of neural activation associated with 100 days of cognitive training. Journal of Cognitive Neuroscience, 31(6), 808-820.

Sporns, O. (2014). Contributions and challenges for network models in cognitive neuroscience. Nature Neuroscience, 17(5), 652.

Sporns, O. (2018). Graph theory methods: applications in brain networks. Dialogues in Clinical Neuroscience, 20(2), 111-121.

Sripada, C., Taxali, A., Angstadt, M., \& Rutherford, S. (2020). Boost in test-retest reliability in resting state fMRI with predictive modeling. BioRxiv, 796714.

Strangman, G. E., O'Neil-Pirozzi, T. M., Supelana, C., Goldstein, R., Katz, D., \& Glenn, M. B. (2010). Regional brain morphometry predicts memory rehabilitation outcome after traumatic brain injury. Frontiers in Human Neuroscience, 4, 182.

Strobach, T., \& Karbach, J. (Eds.). (2020). Cognitive training: an overview of features and applications (2nd ed.). Springer.

Sui, J., Jiang, R., Bustillo, J., \& Calhoun, V. (2020). Neuroimaging-based individualized prediction of cognition and behavior for mental disorders and health: methods and promises. Biological Psychiatry.

Suo, C., Singh, M. F., Gates, N., Wen, W., Sachdev, P., Brodaty, H., et al. (2016). Therapeutically relevant structural and functional mechanisms triggered by physical and cognitive exercise. Molecular Psychiatry, 21(11), 1633-1642.

Supekar, K., Swigart, A. G., Tenison, C., Jolles, D. D., Rosenberg-Lee, M., Fuchs, L., \& Menon, V. (2013). Neural predictors of individual differences in response to math tutoring in primary-grade school children. Proceedings of the National Academy of Sciences, 110(20), 8230-8235. https://doi.org/10.1073/pnas.1222154110.

Takeuchi, H., Sekiguchi, A., Taki, Y., Yokoyama, S., Yomogida, Y., Komuro, N., ... Kawashima, R. (2010). Training of working memory impacts structural connectivity. The Journal of Neuroscience, 30(9), 3297-3303. https://doi.org/10.1523/jneurosci.4611-09.2010.

Takeuchi, Hikaru, Taki, Y., Nouchi, R., Hashizume, H., Sekiguchi, A., Kotozaki, Y., ... Kawashima, R. (2013). Effects of working memory training on functional connectivity and cerebral blood flow during rest. Cortex, 49(8), 2106-2125. https://doi.org/10.1016/j.cortex. 2012.09.007.

Taya, F., Sun, Y., Babiloni, F., Thakor, N., \& Bezerianos, A. (2015). Brain enhancement through cognitive training: a new insight from brain connectome. Frontiers in Systems Neuroscience, 9, 44. https:// doi.org/10.3389/fnsys.2015.00044.

Ventura-Campos, N., Sanjuan, A., Gonzalez, J., Palomar-Garcia, M.-A., Rodriguez-Pujadas, A., Sebastian-Galles, N., ... Avila, C. (2013). Spontaneous brain activity predicts learning ability of foreign sounds. The Journal of Neuroscience, 33(22), 9295-9305. https:// doi.org/10.1523/JNEUROSCI.4655-12.2013.

Verghese, A., Garner, K. G., Mattingley, J. B., \& Dux, P. E. (2016). Prefrontal cortex structure predicts training-induced improvements 
in multitasking performance. Journal of Neuroscience, 36(9), 2638 2645.

Vo, L. T., Walther, D. B., Kramer, A. F., Erickson, K. I., Boot, W. R., Voss, M. W., ... \& Simons, D. J. (2011). Predicting individuals' learning success from patterns of pre-learning MRI activity. PLoS One, 6(1), e16093.

Wenger, E., Brozzoli, C., Lindenberger, U., \& Lövdén, M. (2017). Expansion and renormalization of human brain structure during skill acquisition. Trends in Cognitive Sciences, 21(12), 930-939. https:// doi.org/10.1016/j.tics.2017.09.008.

Wiemers, E. A., Redick, T. S., \& Morrison, A. B. (2019). The influence of individual differences in cognitive ability on working memory training gains. Journal of Cognitive Enhancement, 3(2), 174-185. https://doi.org/10.1007/s41465-018-0111-2.

Wig, G. S. (2017). Segregated systems of human brain networks. Trends in Cognitive Sciences, 21(12), 981-996. https://doi.org/10.1016/j. tics.2017.09.006.

Wolf, D., Fischer, F. U., Fesenbeckh, J., Yakushev, I., Lelieveld, I. M., Scheurich, A., ... Fellgiebel, A. (2014). Structural integrity of the corpus callosum predicts long-term transfer of fluid intelligencerelated training gains in normal aging. Human Brain Mapping, 35(1), 309-318. https://doi.org/10.1002/hbm.22177.
Woo, C. W., Chang, L. J., Lindquist, M. A., \& Wager, T. D. (2017). Building better biomarkers: brain models in translational neuroimaging. Nature Neuroscience, 20(3), 365.

Yamashita, M., Kawato, M., \& Imamizu, H. (2015). Predicting learning plateau of working memory from whole-brain intrinsic network connectivity patterns. Scientific Reports, 5, 7622. https://doi.org/ 10.1038/srep07622.

Zinke, K., Zeintl, M., Rose, N. S., Putzmann, J., Pydde, A., \& Kliegel, M. (2014). Working memory training and transfer in older adults: effects of age, baseline performance, and training gains. Developmental Psychology, 50(1), 304. https://doi.org/10.1037/ a0032982.

Zuo, X. N., \& Xing, X. X. (2014). Test-retest reliabilities of resting-state FMRI measurements in human brain functional connectomics: a systems neuroscience perspective. Neuroscience \& Biobehavioral Reviews, 45, 100-118.

Publisher's Note Springer Nature remains neutral with regard to jurisdictional claims in published maps and institutional affiliations. 